\section{Bias in CFTR screening panels}

To the Editor: The comparison of CFTR mutation screening panels with population frequencies using data from the Exome Aggregation Consortium carried out by Lim and colleagues ${ }^{1}$ highlights the continued gap in annotation of variation in CFTR. The Exome Aggregation Consortium cohort presents a much more ethnically and geographically comprehensive sampling of individuals than the collections of cystic fibrosis (CF) patients from which mutation screening panels are derived. The authors therefore conclude that the sensitivity of CF screening could be improved by replacing panels with exome sequencing.

Sequencing has an important role in CF patients with defined phenotypes and will enhance understanding of the role of CFTR variants in non-European individuals who present with phenotypic features not readily recognized as CF.

However, we feel that sequencing is not a better way to achieve the goals of carrier screening. Carrier screening has traditionally focused on variants that have known, life-threatening consequences in order to enable unaffected heterozygous patients to make informed reproductive decisions. ${ }^{2}$ Sequencing and the potential identification in screened individuals of uncharacterized variants that may have reduced penetrance promotes selection against relatively benign phenotypes along with true disease-causing conditions. Furthermore, the use of computational mutation prediction scores from PolyPhen-2 and PROVEAN are too imprecise and undervalidated for clinical decision making because they lack specificity. ${ }^{3}$ For example, a variant with a slight effect on reproductive fitness (such as those associated with only obstructive azoospermia) is categorized as pathogenic in the same fashion as a variant that is fully penetrant for life-shortening CF. Therefore, the authors may be overestimating the number of $\mathrm{CF}$-causing mutations that

\section{Response to Sosnay et al.}

To the Editor: We thank Drs Sosnay et al. ${ }^{1}$ for their thoughtful comments. Their commitment to CFTR research continues to add valuable clarity to the clinical and functional consequences of CFTR genetic variation.

We set out to quantitate what we as a genetics community have been aware of for decades: cystic fibrosis carrier screening does not adequately identify reproductive risk among nonEuropean populations. Current "pan-ethnic" carrier screens stipulate as much, ${ }^{2}$ and yet CFTR is among the most rigorously studied and well-covered disease genes. go undetected on traditional carrier screens. Indeed, the lack of information to adequately counsel an asymptomatic carrier with an uncharacterized variant is an important unmet challenge in implementing personalized medicine. ${ }^{4}$

\section{ACKNOWLEDGMENTS}

The CFTR2 project is funded by unrestricted educational grants from the US CF Foundation, Vertex Pharmaceuticals, and Sequenom, Inc.

\section{DISCLOSURE}

The authors declare no conflict of interest.

Patrick R. Sosnay, $M D^{1}$, Carlo Castellani, $M D^{2}$, Christopher $M$. Penland, PhD', Johanna M. Rommens, PhD', Michelle Lewis, $M D$, JD ${ }^{1}$, Karen S. Raraigh, $M G C^{1}$, Mary Corey, PhD and Garry R. Cutting, $M D^{1}$

${ }^{1}$ McKusick-Nathans Institute of Genetic Medicine, Johns Hopkins University, Baltimore, Maryland, USA; ${ }^{2}$ Cystic Fibrosis Center, Azienda Ospedaliera Universitaria Integrata, Verona, Italy; ${ }^{3}$ Cystic Fibrosis Foundation, Bethesda, Maryland, USA; ${ }^{4}$ Program in Genetics and Genome Biology, The Hospital for Sick Children, Toronto, Ontario, Canada; ${ }^{5}$ Program in Child Evaluative Health Sciences, The Hospital for Sick Children, Toronto, Ontario, Canada. Correspondence: Patrick R. Sosnay (psosnay@jhmi.edu)

\section{REFERENCES}

1. Lim RM, Silver AJ, Silver MJ et al. Targeted mutation screening panels expose systematic population bias in detection of cystic fibrosis risk. Genet Med; e-pub ahead of print 16 April 2015.

2. Grody WW, Thompson BH, Gregg AR et al. ACMG position statement on prenatal/preconception expanded carrier screening. Genet Med 2013;15: 482-483.

3. Dorfman $R$, Nalpathamkalam $T$, Taylor $C$ et al. Do common in silico tools predict the clinical consequences of amino-acid substitutions in the CFTR gene? Clin Genet 2010;77:464-473.

4. Rehm HL, Berg JS, Brooks LD. ClinGen-the clinical genome resource. N Engl J Med 2015:372:2235-2242.

Advance online publication 29 October 2015. doi:10.1038/gim.2015.105

New pathogenic variants are currently added to ClinVar on a monthly basis, and affected children continue to be diagnosed with novel variant combinations. Thus, clinically-based pathogenic data sets will always be incomplete. We believe it is our obligation as genetic researchers to acknowledge this complex reality while utilizing the full force of scientific progress to illuminate disease risk in all populations. One crucial step in this direction is the adoption of exome sequencing as the standard for reproductive risk analysis. Exome sequencing provides an opportunity to level the analytic playing field. It eschews the restrictions and population bias inherent in targeted mutation testing. Further, exome sequencing sets the foundation for a sophisticated interpretation of genetic variation and reproductive disease risk. 